\title{
Reflets
}

Revue d'intervention sociale et communautaire

\section{Les services en santé mentale pour les jeunes francophones de la région d'Ottawa}

\section{Marie-Pier Audet et Marie Drolet}

Volume 20, numéro 2, automne 2014

URI : https://id.erudit.org/iderudit/1027592ar

DOI : https://doi.org/10.7202/1027592ar

Aller au sommaire du numéro

Éditeur(s)

Reflets, Revue d'intervention sociale et communautaire

ISSN

1203-4576 (imprimé)

1712-8498 (numérique)

Découvrir la revue

Citer cet article

Audet, M.-P. \& Drolet, M. (2014). Les services en santé mentale pour les jeunes francophones de la région d'Ottawa. Reflets, 20(2), 190-200.

https://doi.org/10.7202/1027592ar d'utilisation que vous pouvez consulter en ligne. 


\title{
Les services en santé mentale pour les jeunes francophones de la région d'Ottawa
}

\author{
Marie-Pier Audet \\ Travailleuse sociale en milieu scolaire an Conseil des écoles publiques de \\ l'Est de l'Ontario \\ Marie Drolet, T.S., Ph. D., \\ Professeure titulaire, École de service social, Université d'Ottawa
}

\section{Introduction}

La recherche en santé mentale chez les jeunes a pris de l'ampleur au cours des dernières décennies (Aïach, 2009; Centre d'excellence provincial au CHEO en santé mentale des enfants et des adolescents (CEPSM), 2010). Bien que les champs de la psychologie et de la psychiatrie étudient depuis plus d'un siècle les troubles de santé mentale qui touchent les adultes (Barlow et Durand, 2007), des recherches plus récentes démontrent que la plupart des problèmes et des troubles de santé mentale vécus par cette population débutent durant l'enfance ou l'adolescence (Kutcher, et collab., 2010).

Le présent article porte sur les problèmes de santé mentale chez les jeunes, et plus particulièrement sur les services en santé mentale, leur accès, et l'usage qu'en font les jeunes francophones vivant en situation minoritaire dans la région d'Ottawa. Il découle du mémoire de maitrise en service social de la première auteure, Comprendre l'histoire des jeunes pour pouvoir mieux les aider : exploration des trajectoires et usages de services en santé mentale d'adolescentes et 
de préadolescents-es francophones en situation minoritaire dans la région d'Ottawa. Ce mémoire résulte d'entrevues menées auprès de sept parents de neuf jeunes francophones qui ont des problèmes de santé mentale. Nous présentons brièvement la problématique de santé mentale chez les enfants et les adolescentes ou adolescents canadiens de même qu'un état de leur accès à des services en santé mentale. Certains des résultats obtenus dans le cadre du mémoire complètent cet article.

\section{Problèmes de santé mentale versus troubles de santé mentale}

Alors que la vision médicale ou psychiatrique existe depuis longtemps, plusieurs chercheurs adoptent depuis peu une vision plutôt «sociale » ou «systémique » pour étudier la problématique de la santé mentale et pour agir sur celle-ci (Landry, 2008; Aïach, 2009). C'est qu'il existe tout un processus chez une personne réagissant à l'environnement et aux événements qu'elle rencontre au cours de sa vie. Ces difficultés émotives et psychiques se traduisent par différents symptômes ou réactions qu'on qualifie de "problèmes de santé mentale » et éventuellement, de "troubles de santé mentale " (Landry, 2008; Aïach, 2009; Suissa, 2009).

Comme la médicalisation exerce un grand pouvoir dans la société occidentale, certaines personnes bénéficieront de services spécifiques en santé mentale seulement si elles ont un diagnostic posé par un médecin. Cependant, certains services se dissocient des diagnostics de santé mentale en s'adressant à toute personne vivant des difficultés sur le plan émotionnel ou psychique (qu'on caractériserait ici de "problèmes de santé mentale "). Cette distinction aide à clarifier comment ces deux termes sont utilisés dans cet article, et pourquoi ni l'un ni l'autre n'a été exclu dans l'échantillon de jeunes faisant partie de notre étude. Un individu peut présenter des "problèmes de santé mentale " et obtenir un diagnostic de trouble mental (TM) de la part d'un médecin, alors qu'un autre peut présenter des "problèmes de santé mentale » sans recevoir de diagnostic. 
Selon la vision médicale, une "maladie mentale " ou un " trouble mental " est défini comme étant "un problème qui répond aux critères de diagnostic du DSM-IV en matière de TM ou d'abus de substance, incluant la présence de niveaux significatifs de symptomatologie et un déficit du fonctionnement " (CEPSM, 2010, p.12). La schizophrénie, la dépression majeure, l'anxiété généralisée et la bipolarité sont quelques exemples de maladies mentales.

Les problèmes de santé mentale sont définis comme "des difficultés qui pourraient signifier des signes ou symptômes précoces de troubles mentaux (ou comportementaux ou émotionnels), mais ne sont pas fréquents ni assez graves pour correspondre aux critères de diagnostic" (CEPSM, 2010, p.12). Un problème de cette nature pourrait se manifester notamment par les sentiments de mal-être, de tristesse ou d'anxiété et par des problèmes de concentration. Entre autres, un problème de santé mentale pourrait représenter tout état de mal-être manifesté par une personne, sans pour autant qu'un diagnostic y soit apposé.

Quant à l'ampleur de la problématique de santé mentale chez les enfants et les adolescentes ou adolescents canadiens, les études indiquent que de 15 à $21 \%$ d'entre eux souffrent de troubles de santé mentale qui causent d'importants symptômes ou des difficultés de fonctionnement (Waddell, et collab., 2007; Kutcher et McDougall, 2009; ministère des Services à l'enfance et à la jeunesse de l'Ontario (MSJE), 2010).

\section{État de la situation : les services en santé mentale offerts aux jeunes Canadiennes et Canadiens présentant des problèmes de santé mentale}

Même si nous savons que des problèmes de santé mentale peuvent s'aggraver si rien n'est fait pour y remédier et que de nombreuses recherches identifient les interventions nécessaires pour tenter d'agir sur la situation, il existe toujours un manque de services appropriés s'adressant aux problèmes de santé mentale chez les 
jeunes au Canada (CEPSM, 2010; Kutcher, et collab., 2010; MSEJ, 2010). Cette réalité, qui est d'autant plus tangible en Ontario, touche aussi la région d'Ottawa (CEPSM, 2010; Kutcher, et collab., 2010; MSEJ, 2010). En fait, Kutcher, et collab. (2010) suggèrent que seulement 20 à $40 \%$ des jeunes Canadiennes ou Canadiens ayant besoin de services en santé mentale les reçoivent véritablement. Un temps d'attente important retarde leur accès à de tels services (CEPSM, 2010; Kutcher, et collab., 2010; MSJE, 2010), lesquels s'avèrent limités par un manque de fonds ${ }^{1}$ et de main-d'œuvre spécialisée ${ }^{2}$ plutôt que par l'intensité des besoins à satisfaire chez les jeunes (MSJE, 2010). Par ailleurs, le CEPSM avance que la fragmentation des services pose des défis majeurs à leur efficacité. En effet, " des différences disciplinaires et des liens cliniques faibles entre les services de spécialiste, les soins de première ligne et les programmes communautaires aboutissent à un manque de collaboration" (CEPSM, 2010, p.20).

Pour ajouter à la complexité de la problématique d'accès aux services en santé mentale, la situation peut être encore plus difficile pour les jeunes francophones en situation minoritaire ${ }^{3}$ qui parfois ne peuvent pas bénéficier de services dans leur langue. Alors qu'il existe à priori un problème d'accès aux services de santé mentale, les études démontrent un manque de recherche en ce qui concerne l'accès aux services en français pour cette tranche de population (Levesque, 2005; Forgues, et collab., 2009). Parmi les parents interviewés dans le cadre de notre mémoire, six parents ont mentionné qu'ils étaient plus à l'aise de recevoir des services dans leur langue première, le français. Or, à l'exception de la mère d'une adolescente, tous ont mentionné avoir reçu au moins un service en langue anglaise. À ce propos, une étude de Madoc-Jones (2004) et un rapport de Santé Canada (2007) démontrent l'importance particulière pour les individus ayant des problèmes de santé mentale de pouvoir s'exprimer dans leur langue première lorsqu'ils reçoivent des services d'évaluation, d'intervention, de traitement ou de réhabilitation. Une difficulté de communication pourrait inévitablement mener à un mauvais diagnostic, à une difficulté de compréhension vis-à-vis du traitement, de l'intervention à entreprendre, ainsi que des demandes de la professionnelle 
ou du professionnel en ce qui concerne les modifications de comportements ou d'habitudes de vie.

\section{Le rôle du travail social en milieu scolaire et du Centre psychosocial d'Ottawa dans l'accès aux services en santé mentale pour les jeunes de la région d'Ottawa}

Cette section présente certains résultats de notre mémoire qui découlent des entrevues qualitatives réalisées au printemps 2013 auprès de sept parents de trois préadolescents et cinq adolescentes francophones, âgés de 10 à 16 ans, qui présentent un ou plusieurs problèmes de santé mentale, et qui ont reçu des services en santé mentale au cours des dernières années. Cet échantillon de répondants volontaires a été recruté au Centre psychosocial, un centre francophone de santé mentale pour enfants, adolescentes ou adolescents et familles, de même qu'au Conseil des écoles publiques de l'Est de l'Ontario (CEPEO). Nous y ajoutons des réflexions liées aux expériences de stage et de travail actuel de la première auteure en tant que travailleuse sociale au sein du CEPEO.

\section{Temps d'attente versus rapidité des services}

Cinq parents interviewés sur sept ont mentionné avoir eu à composer avec un temps d'attente important pour que leur enfant reçoive les services sociaux et de santé mentale nécessaires. Cependant, six parents mentionnent avoir été satisfaits et même surpris de la vitesse de certains services reçus. En fait, cette satisfaction est en grande partie attribuée au Centre psychosocial où, selon le témoignage de leurs parents, cinq jeunes ont été rencontrés dans un intervalle de quelques semaines après avoir démontré un urgent besoin d'aide. Une autre mère mentionne que le Centre psychosocial a pu offrir des services à sa fille dans 
un délai plus bref qu'un autre service d'urgence psychosociale à Ottawa. Cette réalité démontre bien le rôle important que joue le Centre psychosocial auprès des jeunes francophones de la région d'Ottawa qui présentent des problèmes de santé mentale.

\section{Flexibilité et disponibilité des services}

Selon les témoignages recueillis, la satisfaction des parents à l'égard des services en santé mentale s'explique aussi par la flexibilité et la disponibilité manifestées par les intervenantes ou intervenants du Centre psychosocial et par les travailleuses ou travailleurs sociaux en milieu scolaire. Une mère mentionne qu'une intervenante du Centre psychosocial s'est déplacée à plusieurs reprises à l'école de sa fille pour la rencontrer d'urgence.

Dans cette dernière situation, le Centre psychosocial a fait preuve de la flexibilité d'horaire que peut en principe offrir aux jeunes le travail social à l'école. En effet, puisque des travailleuses et travailleurs sociaux sont sur place dans les écoles, ils sont facilement accessibles pour les enfants et les jeunes qui manifestent un besoin immédiat. Entre autres, si un élève se retrouve en situation de crise, la travailleuse ou le travailleur social en milieu scolaire est sur place et peut intervenir rapidement. Dans ce même ordre d'idées, les résultats d'analyse du mémoire démontrent que parmi les neuf enfants des parents interviewés, huit ont reçu des services de la part de travailleuses ou travailleurs sociaux en milieu scolaire. Pour cinq de ces jeunes, cette aide a été la première parmi les services sociaux et de santé mentale qu'ils ont reçus par la suite. Cela dit, le caractère particulier du travail social en milieu scolaire s'explique par son statut à la fois " professionnel » et faisant partie intégrante du contexte scolaire, milieu de vie où les jeunes passent notamment une grande partie de leur semaine.

Pour ce qui est de l'importance des services en français en milieu minoritaire, comme le Centre psychosocial offre des services en santé mentale à la communauté francophone et que les conseils scolaires francophones embauchent uniquement des travailleuses et des travailleurs sociaux francophones, ces deux 
services sont jugés par les parents comme étant très précieux pour les jeunes francophones de la région qui sont confrontés à des problèmes de santé mentale.

\section{Fragmentation versus collaboration entre les services}

Plusieurs articles publiés décrivent la fragmentation des services comme constituant un obstacle crucial à l'accès aux soins en santé mentale pour les jeunes (CEPSM, 2010; MSEJ, 2010). Cette réalité est présente dans le discours de cinq des sept parents interviewés au sujet des divers services en santé mentale dans la région. Cette fragmentation se manifeste par un manque de communication et de collaboration entre organismes, par un refus de services malgré la demande de quelques professionnelles ou professionnels ou par une incohérence entre les évaluations et diagnostics de différents professionnelles ou professionnels.

Par ailleurs, le travail social en milieu scolaire doit en principe faire preuve de collaboration avec les services externes en santé mentale auxquels il réfere les jeunes. Les travailleuses ou travailleurs sociaux jouent un rôle clé au sein du personnel scolaire en ce qui a trait à la santé mentale chez les élèves et à l'aiguillage vers les organismes communautaires (Drolet, Dubois et Zloteanu, 2008). Ce sont eux qui évaluent les besoins des jeunes ou ceux des familles et qui font appel aux services externes avec lesquels ils collaborent pour assurer une prise en charge qui leur soit adaptée.

Selon les témoignages des parents interviewés, le Centre psychosocial constitue un partenaire extrêmement important pour les jeunes de la région. La collaboration entre les travailleuses ou travailleurs sociaux en milieu scolaire et les intervenantes ou intervenants du Centre psychosocial se fait aisément et, vu le petit nombre d'intervenants francophones dans la région, leur collaboration est particulièrement chaleureuse et s'appuie sur l'entraide mutuelle. 
Cette réalité va dans le sens des efforts mis en place par un des principes du Cadre stratégique ontarien des services de santé mentale aux enfants et aux jeunes du MSEJ (2010) qui cherche à améliorer la coordination dans les secteurs offrant des services en santé mentale pour l'enfance et la jeunesse, de même que la collaboration entre ces secteurs. Elle est également mise en valeur par le contenu du rapport du CEPSM (2010) qui déplore une fragmentation des services en santé mentale pour les jeunes. En contrepartie, le travail social en milieu scolaire ainsi que le Centre psychosocial constituent d'excellents exemples de collaboration dans le domaine des services en santé mentale pour les enfants et les adolescentes ou adolescents francophones de la région d'Ottawa.

\section{Conclusion}

Pour conclure, il importe de souligner à nouveau que les recherches portant sur la santé mentale des jeunes augmentent depuis quelque temps au Canada. Plusieurs rapports et articles déplorent un manque de services pour venir en aide à cette tranche de la population (Reid et Brown, 2008; CEPSM, 2010; Dion, et collab., 2010). En ce qui concerne les jeunes francophones ayant des problèmes de santé mentale, la situation se présente de façon encore plus complexe. Bien que peu de recherches portent spécifiquement sur les services de santé mentale en français pour les jeunes francophones, des faits inquiétants se dégagent des propos des parents interviewés dans le cadre du mémoire dont découle le présent article : alors que six des sept parents ont mentionné qu'ils étaient plus à l'aise de recevoir des services dans leur langue première, soit le français, tous sauf un parent ont mentionné avoir reçu au moins un service en langue anglaise.

En revanche, malgré les lacunes dans les services, les résultats de notre recherche démontrent que certains services déjà en place sont remarquables. En nous basant sur les résultats des entrevues, nous pouvons souligner le rôle important que jouent le travail social en milieu scolaire et le Centre psychosocial dans l'accès 
et l'usage de services en santé mentale des jeunes francophones de la région d'Ottawa. Ces deux services se démarquent par leur rapidité de réponse, leur flexibilité, leur disponibilité et leur aptitude à collaborer.

Cependant, malgré la satisfaction des parents interviewés à l'égard des services reçus, il ne faut pas oublier que ces deux services ont également des listes d'attentes. La grande rapidité avec laquelle quatre jeunes parmi notre échantillon de recherche ont été rencontrés par une travailleuse ou un travailleur social en milieu scolaire ou par le Centre psychosocial s'explique par le fait qu'ils manifestaient des tendances suicidaires. Cette réalité reflète celle des résultats d'étude du CEPSM (2010) et de Dion, et collab. (2010) démontrant que les services sont davantage disponibles lorsque la famille est en situation de crise.

Il est clair que le manque d'accès aux services est en grande partie causé par le manque de fonds. Pour cette raison, il est important de poursuivre les recherches dans le but de connaitre et de démontrer quels sont les besoins en matière de services dans notre communauté francophone. À tout le moins, le présent article a le mérite de souligner le rôle important que jouent le Centre psychosocial et le travail social en milieu scolaire pour les jeunes francophones de la région d'Ottawa vivant en situation minoritaire et présentant des problèmes de santé mentale.

En tant que récipiendaire de la Bourse Roland Lecomte, Marie-Pier Audet remercie l'Association des travailleuses et travailleurs sociaux de l'Ontario et le Consortium national de formation en santé (CNFS)Volet Université d'Ottawa pour leur généreuse contribution financière à la réalisation de cette étude. Elle veut également souligner le soutien financier du CNFS-Secrétariat national au projet de recherche global auquel est lié son mémoire.

\section{Notes}

1 Reid et Brown (2008) suggèrent un financement inadéquat du système comme représentant un obstacle à l'accès.

2 «Les recherches sur les obstacles à l'accès soulignent des inquiétudes croissantes concernant une crise de main-d'œuvre dans les professions de la santé mentale pour les enfants, particulièrement 
en région rurale. Les défis dans ce domaine incluent le recrutement et la rétention du personnel, en plus de la prestation de services de formation accessibles et efficaces dans les domaines de la formation initiale et du perfectionnement" (CEPSM, 2010, p.20).

3 Les populations francophones en situation minoritaire sont définies comme les populations francophones du Canada, vivant à l'extérieur du Québec (CNFS et SSF, 2010).

\section{Bibliographie}

AÏACH, Pierre (2009). "L'irrésistible expansion du champ de la santé », dans H. Sanni Yaya, Pouvoir médical et santé totalitaire: Conséquences socio-anthropologiques et éthiques, Québec, Québec, Les Presses de l’Université Laval, p. 81-98.

BARLOW, David H., et Vincent M. DURAND (2007). « Comprendre la psychopathologie », dans David H. Barlow et Vincent M. Durand, Psychopathologie, Une perspective multidimensionnelle, Bruxelles, Collection Ouvertures Psychologiques, De Boeck, p. 2-21.

CENTRE D'EXCELLENCE PROVINCIAL AU CHEO EN SANTÉ MENTALE DES ENFANTS ET DES ADOS (CEPSM) (2010). L'accès et les temps d'attente en santé mentale des enfants et des adolescents : Document d'information, document soumis à l'Institut du développement et de la santé des enfants et des adolescents.

CONSORTIUM NATIONAL DE FORMATION EN SANTÉ ET SOCIÉTÉ SANTÉ EN FRANÇAIS (CNFS et SSF) (2010). La santé des francophones en situation minoritaire : Un urgent besoin de plus d'informations pour offrir de meilleurs services : Sommaire, Ottawa, Ontario, Consortium national de formation en santé; Société Santé en français.

DION, Jacinthe, et collab. (2010). "Evaluating crisis intervention services for youth within an emergency department: A view from within ", Child Care in Practice, Vol.16, N³, p. 241-256.

DROLET, Marie, Madeleine DUBOIS et Rodica ZLOTEANU (dires) (2008). Repenser le travail social au sein du Conseil des écoles catholiques de langue française du Centre-Est, Pistes de réflexion et d'orientation, Rapport technique, texte inédit remis au CECLFCE, Ottawa, 85 p.

FOR GUES, Éric, et collab. (2009). De l'émergence à la consolidation: L'état de santé chez les francophones en situation minoritaire, Moncton, Nouveau-Brunswick, Institut canadien de recherche sur les minorités linguistiques.

KUTCHER, Stanley, et Ainslie MCDOUGALL (2009). «Problems with access to adolescent mental health care can lead to dealings with the criminal justice system ", Paediatrics \& Children Health, Vol. 14, p. 15-18.

KUTCHER, Stanley, Mary Jane HAMPTON et Jeffrey WILSON (2010). « Child and adolescent mental health policy and plans in Canada: An analytical review ", Canadian Journal of Psychiatry, 55(2), 100-107.

LANDRY, Balas L. (2008). L'approche systémique en santé mentale, $2^{\mathrm{e}}$ édition, Paramètres, Les Presses de l'Université de Montréal.

LEVESQUE, Annabel (2005). Les adolescentes et les adolescents : une population à risque - La situation des francophones en milieu minoritaire, réf. du 30 juin 2014, Winnipeg, Manitoba, Collège universitaire de Saint-Boniface. http://www.cusb.ca/pdf/rapportderecherche/Santedesados.pdf 
MINISTÈRE DES SERVICES À L'ENFANCE ET À LA JEUNESSE (MSEJ) (2010). Une responsabilité partagée. Cadre stratégique ontarien des services de santé mentale aux enfants et aux jeunes, réf. du 30 juin 2014, Ministère de l'Ontario,

http://www.children.gov.on.ca/htdocs/french/topics/specialneeds/mentalhealth/shared_ responsibility.aspx

REID, Graham, et Judith Bell BROWN (2008). « Money, case complexity, and wait lists: Perspectives on problems and solutions at Children's Mental Health Centers in Ontario ", The Journal of Behavioral Health Services \& Research, Vol. 35, № 3.

SUISSA, Jacob Amnon (2009). "Médicalisation, Ritalin et enjeux sociaux : la performance dans la pilule ", chapitre 14, dans H. S. Yaya, Pouvoir médical et santé totalitaire : Conséquences socioanthropologiques et éthiques, Québec, Les Presses de l'Université Laval, p. 301-317.

WADDELL, Charlotte, et collab. (2007). "Preventing mental disorders in children: A systematic review to inform policy-making ", Canadian Review of Public Health, Vol. 98, N 3, p. 166-173. 